\title{
The Monitoring System of Pumping Station Based on FPGA
}

\author{
Li Chenming ${ }^{1}$, Lin Shuo ${ }^{2}$, Gao Hongmin ${ }^{3}$, Sun liquan ${ }^{4}$ \\ ${ }^{1}$ College of Computer and Information Engineering \\ Hohai University \\ Nanjing, China \\ lichenming55@163.com \\ ${ }^{2}$ College of Computer and Information Engineering \\ Hohai University \\ Nanjing, China \\ linlinle@outlook.com \\ ${ }^{3}$ College of Computer and Information Engineering \\ Hohai University \\ Nanjing, China \\ banjamin2006@163.com \\ ${ }^{4}$ College of Computer and Information Engineering \\ Hohai University \\ Nanjing, China \\ 1176547407@qq.com
}

Keywords- monitoring system; EDA; modular design ;FPGA; GSM;

Abstract- Condition monitoring can greatly reduce the cost of Pumping Station, To have reliable operation its condition must be monitored constantly. the paper presents a monitoring system of Pumping Station based on FPGA. The system uses EDA development software Max + plus II to achieve modular design in FPGA, such as data processing module, pump running status judgment module, pump operation control module, alarm module, etc.. And take GSM communication technology as a system of wireless communication, to complete the data communication between data acquisition part, field equipment and remote monitoring center. Clients can real-time monitoring the operating status of pumping station by remote monitoring center .

\section{INTRODUCTION}

Electronic monitoring system to complete the work of the entire pump station monitoring and abnormal alarm and pump operation control, on the one hand to save manpower and material resources, on the other hand can improve the level of automatic management of pumping stations [1]. EDA as the main way of electronic design technology, widely used in a variety of electronic product design and functional improvement stage, with the characteristics of strong system portability [16]. FPGA read the module saving at EEPROM into the RAM. So you can change the module only need to replace the 
EEPROM on the FPGA. FPGA's feature of thousands of times repeated use to ensure the stability and sustainability of system, and reduce the hardware costs of system upgrades.

For this background, we take the South - to - North Water Diversion as the research object, design an new pumping monitoring architecture based FPGA. Experiments show that, our monitoring system has greatly improve the operation efficiency of the pumping station and realize the goals of high availability, long-term stability and automatic operation of the pumping station equipment.

\section{THE FRAMEWORK DESIGN}

Pump station monitoring system can be divided into water supply pump station monitoring system and drainage pumping station monitoring system according to the pump work style [2]. However, the main target of the system is to achieve the all-weather monitoring and control of the operation status of the pumping station equipment. Through the monitoring of the pump unit and its operating environment, and analyze pump station operation data to ensure the safe operation of the pump station and the surrounding environment, when the pump station running problems, the staff can be found in time, and take the appropriate response measures [3].

Pump station monitoring system with data acquisition and processing functions, monitoring functions, alarm function, communication function, data display storage function, other advanced features .

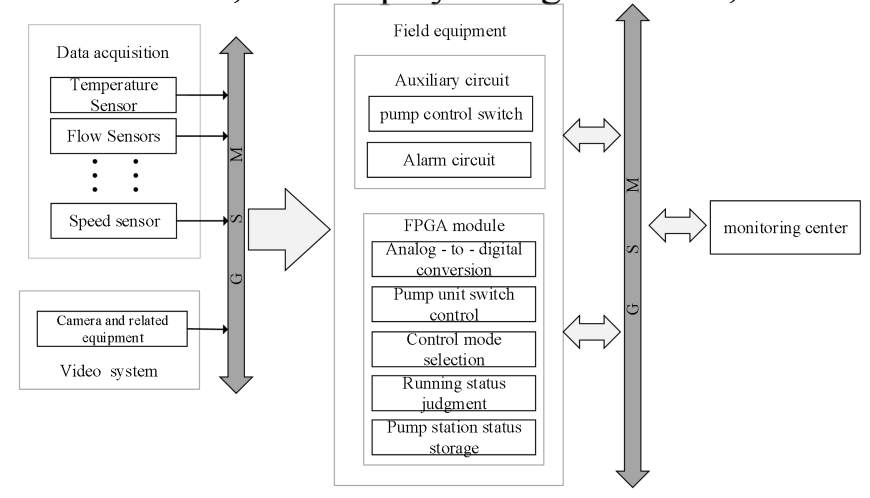

Figure 1. The general design structure of the system.

Pump station monitoring system can be divided into four parts, as shown in Fig.1. The data acquisition part is connected with the field equipment, mainly composed of various sensors and cameras, for collecting various types of equipment operation data and image data [4]. Pump monitoring equipment from a variety of functions of the FPGA module and a variety of control circuits. Wireless communication module complete the data communication between data acquisition part, field equipment and remote monitoring center.

\section{THE DESIGN OF SYSTEMS NETWORK ARCHITECTURE}

\section{A. The Design Offield equipment}

Field monitoring equipment consists of the FPGA modules and control circuits, the need to complete the data processing, the pump running status judgments and data information and status information sent and other functions.

FPGA design in a modular way [5], the design of the advantages of the system can make the overall function of the system and the specific function of the module to achieve the time to shorten the structure of the system architecture, design easier and improve design efficiency. But also in the monitoring system 
based on the existing functions, to facilitate the addition of other functions of the module, the module and the interaction between the module is relatively small, the system with high reliability. FPGA module:

- Data processing: to complete the analog - to - digital conversion of input signal.

- Pump running status judgment module: the module body is a custom comparator, the comparator to complete the data comparison, the module to compare the results to determine the pump running status.

- Pump control module: the custom switch by Verilog programming language, according to determine the output status of the module to control the pump unit switch and alarm module switch..

- Alarm module: including pump abnormal alarm and burglar alarm circuit, control by the FPGA abnormal alarm module.

The auxiliary circuit is composed of pyroelectric detection circuit and switching circuit which control the start and stop of the pump and the alarm circuit. Switch circuit body for the high level control electromagnetic relay, signal issued by the FPGA control switch off.

B. The Design Of GSM Network

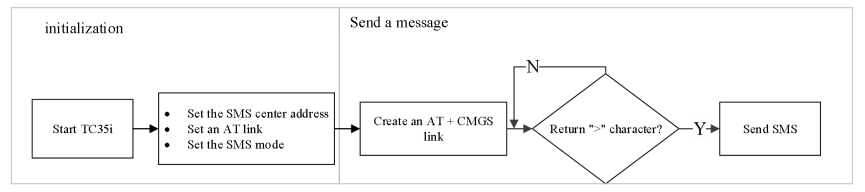

Figure 2. GSM communication flowchart

The monitoring system uses GSM technology as the system communication method. In order to protect the efficiency of data transmission, in the choice of communication modules above or to develop a complete 2G, 3G communication module [6]. The communication module selects Siemens TC35i communication module. Module performance in all aspects, including a SIM application toolkit, the module's data input and output are AT command set control implementation, widely cited in the monitoring industry. TC35i module initialization and send short message flow shown in Figure 2.

C.The Design Of Monitoring software

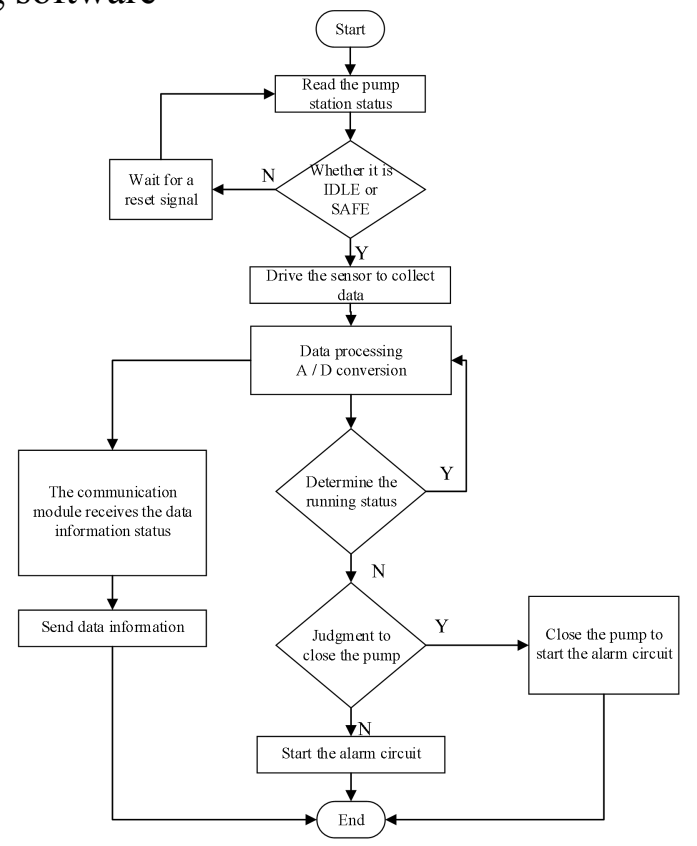

Figure 3. Monitoring system software flowchart 
Pump station monitoring system work flow: the system began to work, read the pump state memory module stored in the running state, if the state is dangerous or alarm state, waiting for the outside world to send system reset command, the system reset. If it is idle or safe state, the system receives the pump motor control mode selection signal; drives the sensor to open the measurement data, the acquired data to complete the analog conversion, the hardware circuit to send data to the communication module. The pump running status judgment module to work, whether the end of the data in the pump station running within the safety parameters, if not in the safe range, to determine the pump running for what state, whether the need to immediately close the pump, you need to start the pump control circuit to close the pump, Start the sound and light alarm circuit; if you do not need to close the pump, only start the alarm circuit. If the data shows that the pump station is running safely, the system processing begins processing the next group of data. The communication module receives the operating status information regardless of the operating state of the pump station. After the communication module completes the reception of the running data and the status information, the data is transmitted to the host computer [7]. The workflow is shown in Figure 3.

\section{SYSTEM DETAILED DESIGN AND IMPLEMENTATION}

This article uses Max + plus II development software [8]. When designing functional modules based on programmable logic devices, the software can achieve a complete design process from design input, electrical component adaptation to module function timing simulation, and also provides designers with a wealth of common electrical components for designers to call, Such as common logic gate components, various types of flip-flops, counters, encoders and decoders.

\section{Implementation of FPGA Function Module}

\section{1) Analog - to - digital conversion module}

The sensor output data is an analog signal, so the A / D converter module is required to convert the input analog signal into a digital signal. AD0809 design for the use of analog-to-digital converter, AD0809 controlled by the FPGA to achieve signal conversion.

Figure 5 ADC08019 module shown, the system clock input CLK is generally 50MHz, ABC_IN [2..0] for the input signal to carry the strobe signal, ABC_OUT is FPGA to ADC0809 channel selection signal, $\mathrm{D}$ [7: 0] is the data that the ADC0809 came after completing the analog-to-digital conversion, DATA_out [7:0] is the output port of the FPGA output to complete the analog-to-digital conversion data. The reset function of the module is determined by the output status of the OE port.

\section{2) Pump running status judgment module}

Field equipment needs to be based on the pump running data to determine the pump running status, control the pump power switch. As shown in the Judge module in Figure 5, DATA_IN: input pump operating status data, SAFE_MAX: there is a monitoring program from the data register pump station safety range reference parameter maximum, DANGER_MIN; input pump station hazard range reference data minimum, Q; Output the comparator ratio after the result as an input signal to the control module.

This module will be trigger if the data input. The module will get the output status data from the A / D conversion control module. The control module controls the input pump station safety range reference data minimum value and the maximum value of the hazard range reference data. Comparator compares the size between the data, and if the input data is less than the maximum value of the safety range, the $Q$ port outputs the binary data "00". If the data is between the maximum value of the safety range and the minimum value of the hazard range, the $Q$ port outputs the binary Data "01", if the maximum value of the hazard range $Q$ port output binary data "10".

3) Auxiliary circuit

After making a judgment on the pump running status, the monitoring system should respond to the operating state, shutting down the pump and enable the alarm circuit when the pump station is at risk, or only enable the alarm circuit. As shown in the Switch module in Figure 5, PUMP_STATUS: Obtain the 
operating status information of the pump from the judgment module, EN: Enable signal for automatic control of the pump, EN_PUMP_CONTROL: Water pump start / stop switch control signal, EN_ALARM: Alarm circuit switch control signal.

First, the module determines whether there is a reset signal input, reset signal is EN_PUMP_CONTROL and EN_ALARM are set to low level. If there is no reset signal, then determine the input pump running status information, "00" shows that the pump station is running normally, the EN_PUMP_CONTROL and EN_ALARM are set to low level. "01" shows that the pump station is running abñormally, but does not need to shut down the pump, the module sets EN_PUMP_CONTRO low, EN_ALARM is high level. "10" indicates that the pump is running abnormally, and the data indicates that the pump continues to run, which may cause damage to the device and the module puts EN_PUMP_CONTROL and EN_ALARM high level.

4) Pump current status latch $\overline{\text { module }}$

The module is uses to store and read the current state of the system and control the sensor to enable when the monitoring software starts working. The actual state machine contains the state: IDLE, SAFE, ALARM, DANGER, the transition between the state shown in Figure 4:

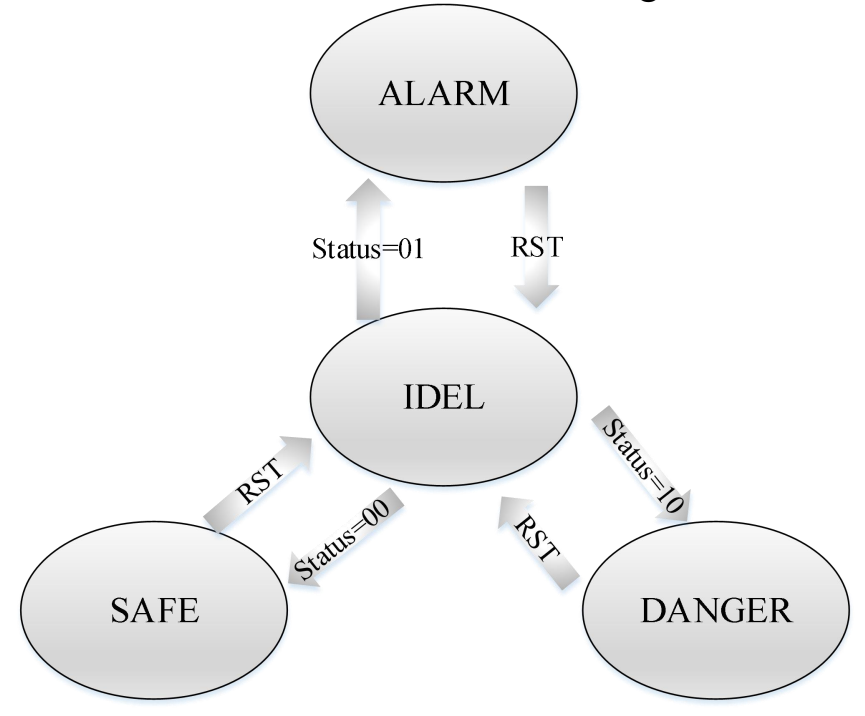

Figure 4. Working state transition map

The current status of the pump station is divided into idle reset status IDEL, safety status SAFE, running abnormal alarm ALARM status and running abnormality, equipment danger DANGER state, state machine according to the input signal to select the current state.

When the system starts working for the first time or the system is reset, the status latch status is IDLE; when the status signal input is "00", the monitor status is SAFE; "01" is the monitor state is ALARM; "10" then the monitor status is DANGER, STATE port outputs the current status.

5) Control mode choose module

Pump station can start and stop with the manual control or automatic control, select the automatic control, the switch will only stop the pump when the problem arise, manual control can be achieved stop the pump at any time base on needs. As shown in the control_choose module in Figure 5:

SIGNAL is the input control mode selection signal, monitoring system with external input control signal, if we choice automatic control, SINGAL received "01" for manual control, "10" for automatic control, EN_SWITCH output high level, control switch circuit control part start working. DISP_WAY is used to control the LED. 


\section{Experimental results}

Combine the FPGA modules above all, as shown in Figure 5:

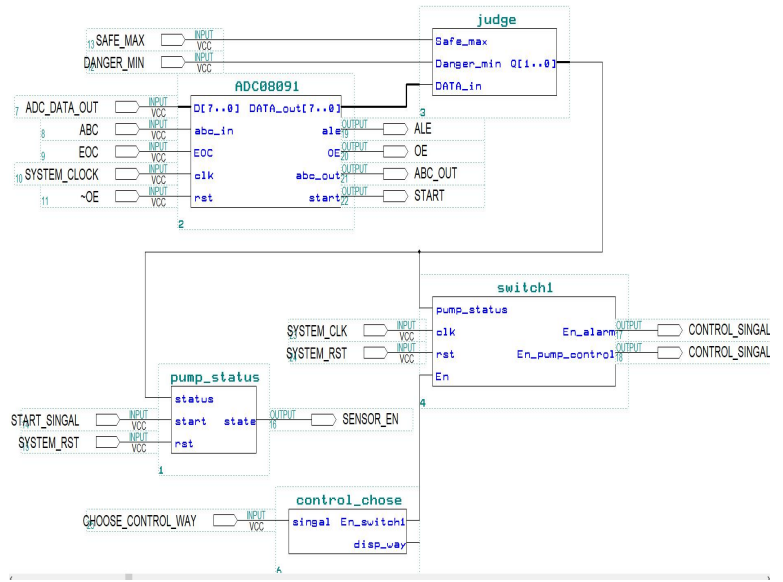

Figure 5. The circuit of FPGA modules

As shown in the table I, enter the 8-bit binary data for comparison, select the input control mode, the judge module to get the results, the switch module according to the control mode and determine the results, the output control signal. As a result of the use of FPGA and GSM, the system has advantages of good real-time, high reliability, stable operation, flexible adjustment, etc..

\section{CONCLUSION}

In reality, many of the monitoring equipment of the pumping station is relatively old, and the lack of effective monitoring of the pumping station unit, resulting in serious problems such as aging of hardware and software, failure rate increases, this paper presents a pump station monitoring system based-FPGA. FPGA functions in a modular way to design, you can add new monitor modules to the system based on the existing system, without having to worry about each other. GSM communication technology as a system of wireless communication, to complete the data communication between data acquisition part, field equipment and remote monitoring center. The system reliability is high, improve the operation efficiency of the pumping station, and realize the high utilization rate, long-term stability and automatic operation of the pumping station equipment.

\section{REFERENCES}

[1] E Pistun, N Yakymchuk. Development of the automatic control system of pumping station for urban water supply[J]. IAPGOS, 2014; 4 (2) : 68-71.

[2] Luo Xinlei, Gao Zhanyi, Feng Guangzhi et al. Study on aging of pumping station engineering [J]. Journal of Hydraulic Engineering, 1997, (05): 43-49.

[3] Huo Ning. Pump station monitoring system and its structure development trend [J]. Hydropower and Pumped Storage, 2004, (04): 81-85.

[4] Guo Jianyong, Zhang Lanzhi,et al.Yang Xiaodong.Pump station monitoring system integrated platform to achieve [J]. Automation and Instrumentation, 2014, (06): 37-40.

[5] Xian Zhimiao. Design and Practice of Digital System for FPGA / CPLD [J]. Journal of Guangxi Normal University(Natural Science Edition), 2005, (01): 38-41. 
[6] Guo Bingjun, Liu Fuyang, Jiang Aiping, Luo Shibo, Yu Jinshou. Application of GSM in Remote Monitoring System of Drainage Pumping Station [J]. Information Technology2004, (05):5-7+66.

[7] Zhao Lin,Study on monitoring system of gate (pump) station in Shandong section of South-to-North Water Diversion Project [D]. Shandong University, 2012.

[8] Che Xuanyu. Two methods of electronic design using MAX + PLUS II[J]. Journal of Lanzhou Institute of Technology ,2004,(02):46-48.

TABLE I. EXPERIMENTAL RESULTS

\begin{tabular}{|c|c|c|c|c|c|c|c|}
\hline \multicolumn{3}{|c|}{ Data input } & \multirow{2}{*}{ Control method } & \multirow{2}{*}{ Judgment } & \multirow{2}{*}{ State machine } & \multicolumn{2}{|c|}{ Switchl } \\
\hline Safe_Max & Danger_Min & $D A T A \_$in & & & & Alarm & Pump_control \\
\hline $0 \times 32$ & $0 \times 3 C$ & $0 \times 2 D$ & & 00 & SAFE & Low level "0" & Low level "0" \\
\hline $0 \times 32$ & $0 \times 3 C$ & $0 \times 37$ & 10 & 01 & ALARM & High level " 1 " & Low level "0" \\
\hline $0 \times 32$ & $0 \times 3 C$ & $0 \mathrm{x} 41$ & & 10 & DANGER & High level "1" & High level "1" \\
\hline $0 \times 32$ & $0 \times 3 C$ & $0 \times 2 D$ & & 00 & SAFE & Low level "0" & \\
\hline $0 \times 32$ & $0 \times 3 C$ & $0 \times 37$ & 01 & 01 & ALARM & High level " 1 " & Low level "0" \\
\hline $0 \times 32$ & $0 \times 3 C$ & $0 \times 41$ & & 10 & DANGER & High level " 1 " & \\
\hline & RST $=" 1 "$ & & ** & $* *$ & IDLE & $* *$ & $* *$ \\
\hline
\end{tabular}

